\title{
1 Systematic mapping of drug metabolism by the human gut microbiome
}

3 Pranatchareeya Chankhamjon ${ }^{1}$, Bahar Javdan ${ }^{1}$, Jaime Lopez ${ }^{2}$, Raphaella Hull1 ,

4 Seema Chatterjee ${ }^{1}$, and Mohamed S. Donia ${ }^{1}$

$6{ }^{1}$ Department of Molecular Biology, Princeton University, Princeton, New Jersey, 08544,

7 USA

8 '2Lewis-Sigler Institute for Integrative Genomics, Princeton University, Princeton, New

9 Jersey, 08544, USA

10

11 Correspondence: donia@princeton.edu

12

13

14

15

16

17

18

19

20

21

22

23

24 
ABSTRACT

The human gut microbiome harbors hundreds of bacterial species with

27 diverse biochemical capabilities, making it one of nature's highest density,

28 highest diversity bioreactors. Several drugs have been previously shown to be

29 directly metabolized by the gut microbiome, but the extent of this phenomenon

30 has not been systematically explored. Here, we develop a systematic screen for

31 mapping the ability of the complex human gut microbiome to biochemically

32 transform small molecules (MDM-Screen), and apply it to a library of 575 clinically

33 used oral drugs. We show that $13 \%$ of the analyzed drugs, spanning 28

34 pharmacological classes, are metabolized by a single microbiome sample. In a

35 proof-of-principle example, we show that microbiome-derived metabolism occurs

36 in vivo, identify the genes responsible for it, and provide a possible link between

37 its consequences and clinically observed features of drug bioavailability and

38 toxicity. Our findings reveal a previously underappreciated role for the gut

39 microbiome in drug metabolism, and provide a comprehensive framework for

40 characterizing this important class of drug-microbiome interactions. 


\section{INTRODUCTION}

The oral route is the most common route for drug administration. Upon exiting

the stomach, drugs can be absorbed in the small and/or large intestine to reach the

51 systemic circulation and eventually the liver, or can be transported there directly via the

52 portal vein. Once at the liver, drugs may be metabolized and secreted back (along with

53 their metabolites) to the intestines through bile, via the enterohepatic circulation ${ }^{1,2}$. Even

54 parenterally administered drugs and their resulting metabolites can reach the intestines

55 through biliary secretion. Therefore, whether prior to or after absorption, most

56 administered drugs will spend a considerable amount of time in the small and large

57 intestines, where trillions of bacterial cells reside and form our human gut microbiome.

58 Despite this fact, and the significant inter-individual variability in both the composition

59 and function of the gut microbiome ${ }^{3}$, we know much less about how our microbiome

60 interacts with drugs than about how our liver interacts with them.

Broadly speaking, there are two main types of interactions that can occur

62 between drugs and the microbiome, which may result in significant effects on drug

63 metabolism, bioavailability, efficacy, and toxicity: direct and indirect interactions.

64 Examples of indirect interactions include the competition between microbiome-derived

65 metabolites and administered drugs for the same host metabolizing enzymes ${ }^{4}$,

66 microbiome effects on the immune system in anticancer immunotherapy ${ }^{5-7}$, microbiome

67 reactivation of secreted inactive metabolites of the $\mathrm{drug}^{8}$, and microbiome overall effects

68 on the levels of metabolizing enzymes in the liver and intestine ${ }^{9}$. Direct interactions

69 between administered drugs and the microbiome include the partial or complete

70 biochemical transformation of a drug into more or less active metabolites by 
71 microbiome-derived enzymes (termed herein: Microbiome-Derived Metabolism, or

72 MDM).

The human gut microbiome harbors hundreds of bacterial species, encoding an

74 estimated 100 times more genes than the human genome ${ }^{10}$. This enormous diversity

75 and richness of genes represent a repertoire of yet-uncharacterized biochemical

76 activities capable of metabolizing ingested chemicals, including both dietary and

77 therapeutic ones ${ }^{11}$. Although MDM has been observed for more than 50 years, and in

78 dozens of examples, this process is still mostly overlooked in the drug development

79 pipeline $2,12-14$. Moreover, studies investigating this process have focused mainly on one

80 bacterium or one drug at a time, and no efforts have been spent to systematically

81 assess the ability of the human gut microbiome to metabolize oral drugs or to develop

82 tools for incorporating this type of analysis into the drug development pipeline. This is

83 owed mainly to the enormous complexity of the microbiome, and to the overwhelming

84 technical challenge of testing hundreds of drugs against thousands of cultured isolates

85 under multiple conditions. Unlike liver-derived metabolism, the lack of a systematic,

86 global, and standardized map of MDM has hindered our ability to reliably predict and

87 eventually interfere with undesired microbiome effects on drug pharmacokinetics and/or

88 pharmacodynamics.

To address this gap in knowledge, we here develop a systematic screen for

90 mapping MDM (MDM-Screen, Fig. 1). Our screen relies on three main arms: i) an

91 optimized batch culturing system for sustaining the growth of complex, personalized,

92 human microbiome-derived microbial communities; ii) a high-throughput analytical

93 chemistry platform for screening hundreds of clinically used small molecule drugs; and 
94 iii) a defined mouse colonization assay for assessing the effect of the microbiome on the

95 pharmacokinetics of selected drugs. Using MDM-Screen with 575 clinically used, orally

96 administered, small molecule drugs, we discovered that $13 \%$ of them can be subject to

97 MDM. As a proof-of-principle example, we selected one of these transformations -

98 MDM deglycosylation of fluoropyrimidines - for further functional investigations. We

99 identify microbiome-derived species and enzymes responsible for this transformation,

100 show that it occurs in vivo in a microbiome-dependent manner, and provide evidence

101 that its consequences may explain outcomes already observed in the clinic. Our screen

102 described here, and the findings obtained from it represent the first systematic map of

103 microbiome-derived metabolism of clinically used drugs, and provide a framework for

104 incorporating an “MDM” module in future drug development pipelines.

105

106

107

108

109

110

111 
<smiles>c1ccccc1</smiles>

Healhy Donor 1

(HD-1)
(1)

ex vivo culture

(2)

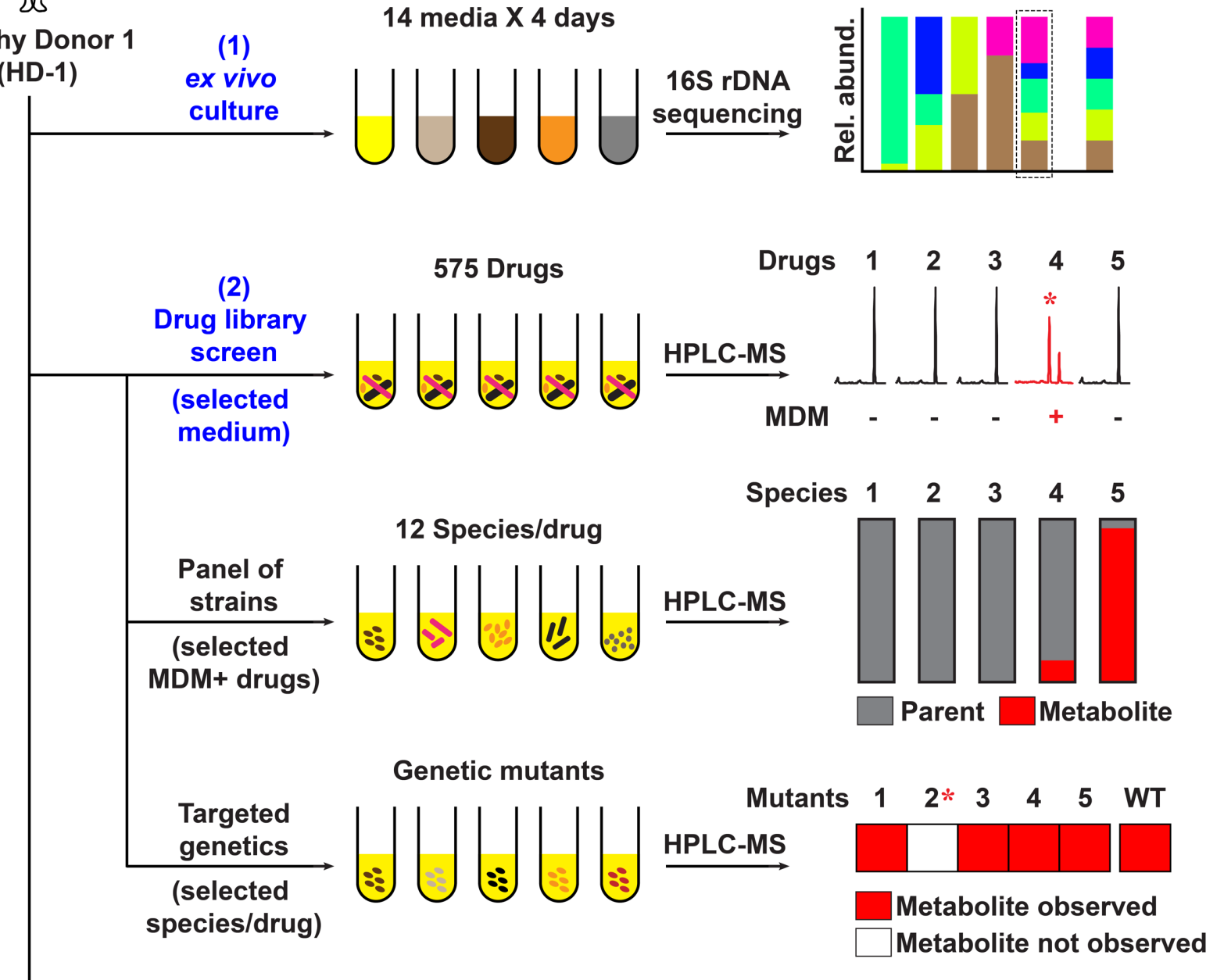

\title{
MDM-Screen
}

Media $\quad \begin{array}{llllll}1 & 2 & 3 & 4 & 5 & \text { HD-1 }\end{array}$

\author{
14 media $X 4$ days
}

(3)

in vivo verification

(selected MDM+ drugs)

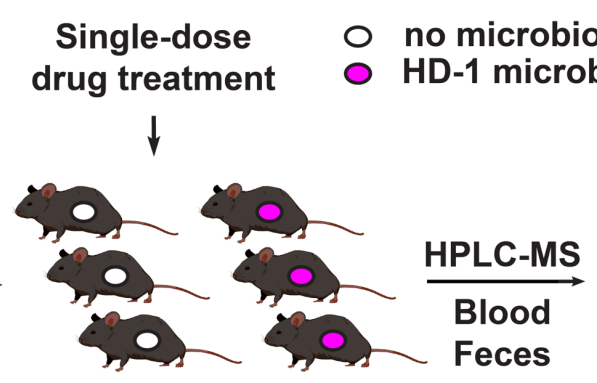

Differential colonization

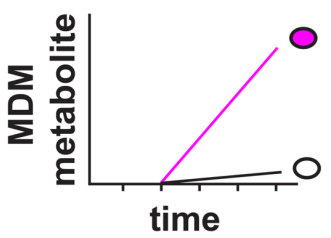

Microbiome-dependent pharmacokinetics 
Figure 1. General approach of MDM-Screen. MDM-Screen is comprised of

121 three arms. (1) An optimized ex vivo culturing model of the gut microbiome in batch

122 format, where a fecal sample from a healthy donor (HD-1) is cultured in 14 different

123 media for 4 days, and the best culturing condition is determined by high-throughput $16 \mathrm{~S}$

124 rDNA amplicon sequencing. (2) A biochemical screen, where the ability of the cultured

125 HD-1 microbiome to metabolize 575 drugs is determined using HPLC-MS. By screening

126 a diverse set of gut isolates, the same platform is used to identify members of the

127 microbiome that may be responsible for specific modifications. Finally, specific genes

128 and enzymes responsible for the modifications are identified by targeted mutagenesis in

129 selected species. (3) For selected MDM cases, a microbiome-dependent

130 pharmacokinetic experiment is performed in mice to assess whether the same drug

131 modification can be observed in vivo. 


\section{RESULTS}

144 An optimized ex vivo culturing model for the human gut microbiome

A major challenge in studying the capacity of the human gut microbiome to

146 metabolize orally administered drugs is the enormous diversity of the bacterial species

147 involved: a typical gut microbiome sample harbors hundreds of species and thousands

148 of strains, many of which are found only in a subset of healthy individuals ${ }^{3,15}$. It is

149 therefore impractical to systematically screen thousands of isolated strains against

150 hundreds of drugs, forcing previous studies to rely mainly on a selected set of

151 representative species. Moreover, gene expression profiles and the significance of a

152 given biochemical transformation may vary dramatically between a monocultured strain

153 and one that is grown in a mixed community. To address these challenges, we sought

154 to develop the first arm of MDM-Screen: an optimized ex vivo culturing system that a)

155 supports the growth of a large proportion of the species from a given microbiome

156 sample in a similar taxonomical composition, and b) is amenable to high-throughput

157 biochemical screens.

Acknowledging the fact that a significant fraction of the community will inevitably

159 evade cultivation efforts, we undertook a systematic approach to identify the medium

160 and culturing period that can support the growth of the maximal number of species in a

161 batch culture of a mixed community. Freshly collected human feces from a healthy

162 donor (referred to as HD-1) were transferred to an anaerobic chamber, suspended in

163 PBS with $0.1 \%$ cysteine, and stored in aliquots of dozens of glycerol stocks. We then

164 started cultures (anaerobic, $37^{\circ} \mathrm{C}$ ) from glycerol-stocked HD-1 in 14 different media,

165 and collected samples daily for 4 days. Finally, we extracted DNA from all cultures, 
amplified the V4 region of the bacterial 16S rRNA gene, and deeply sequenced the amplicons using Illumina (100,000 sequences per sample, on average). From the sequencing results, amplicon sequence variants (ASVs) were inferred using DADA2

169 plugin within QIIME2, and the final taxonomical composition at different levels was 170 determined for each sample using a naive Bayes classifier trained on the Greengenes 171 database ${ }^{16-19}$. We then quantified the differences between the various media and the 172 original fecal sample at both the family level (using the Jensen-Shannon divergence

173 (DJs), a metric that measures the similarity of two distributions), as well as at the single ASV level (to infer the recovery rate of species from the original sample).

176 great level of variation in both the taxonomical composition and diversity between the 177 different media and culturing periods. Some media led to highly diverse communities 178 that captured portions of the original fecal diversity, while others became dominated 179 almost exclusively by a single family. Second, among the 14 media commonly used in 180 cultivation efforts from the human microbiome ${ }^{20}$, we identified one medium, modified 181 Gifu Anaerobic Medium (mGAM), that supported the growth of a bacterial community 182 most similar in composition and diversity to the one observed in HD-1 (Fig. 2a, 183 Supplementary Fig. 1). At the family level, mGAM cultures largely match the 184 composition of HD-1, differing primarily in a commonly observed expansion of the 185 facultative anaerobes, Enterobacteriaceae, at the expense of the obligate anaerobes, 186 Ruminococcaceae. This is likely a result of the inevitable exposure to oxygen during 187 sample handling until delivery to the anaerobic chamber ( $30 \mathrm{~min})^{21}$. Among all tested 188 media, mGAM cultures showed the lowest DJs divergence from HD-1, becoming 
189 increasingly similar to the original sample as growth proceeds (see Supplementary

190 Fig. 1 for the entire four-day time course).

Even at the single ASV level, mGAM cultures capture much of the diversity in

192 HD-1 (mGAM cultures have the highest Shannon diversity across all media, and the

193 closest one to HD-1) (Fig. 2b and Supplementary Fig. 2). In the original fecal sample,

194 there are 33 ASVs present above a relative abundance of 1\%, 26 (79\%) of which are

195 present in mGAM day two culture. Overall, total shared ASVs between the original fecal

196 sample and mGAM day two account for $70 \%$ of the HD-1 composition, indicating that

197 the mGAM culture recapitulates the bulk of the original community. Taken together, and

198 consistent with previous reports showing that mGAM can support the growth of a wide

199 variety of gut microorganisms in monoculture 20,22 , our results establish mGAM day two

200 cultures as a viable ex vivo batch culturing model for the human gut microbiome, where

201 a significant portion of the taxonomical diversity from the original fecal sample can be

202 captured and maintained in a similar composition.

203

204

205

206

207

208

209

210

211 
bioRxiv preprint doi: https://doi.org/10.1101/538215; this version posted February 3, 2019. The copyright holder for this preprint (which was not certified by peer review) is the author/funder, who has granted bioRxiv a license to display the preprint in perpetuity. It is made available under aCC-BY-NC-ND 4.0 International license.

a

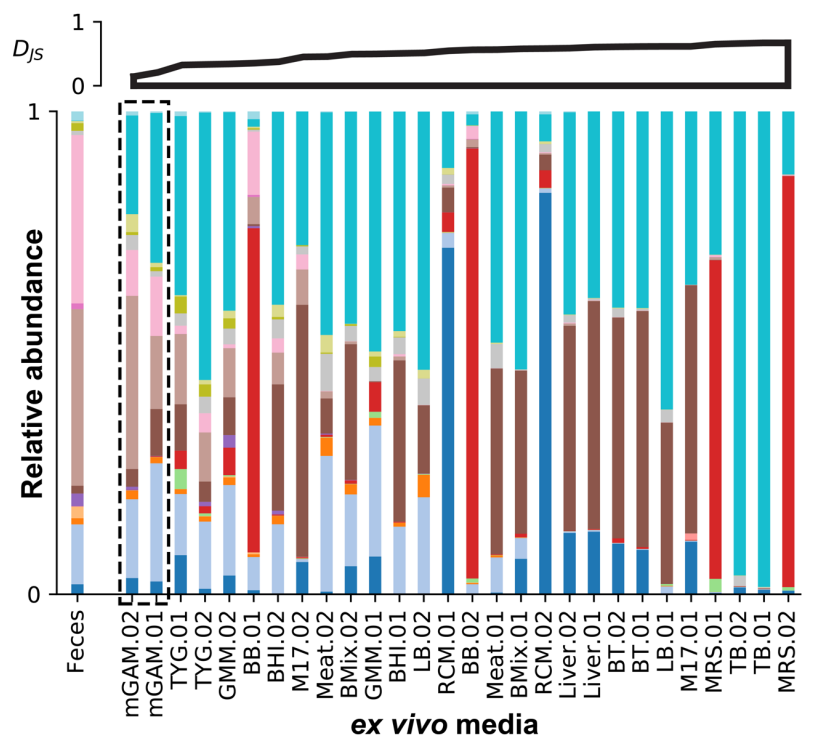

\begin{tabular}{|ll|}
\hline Bifidobacteriaceae & Lachnospiraceae \\
Bacteroidaceae & Peptostreptococcaceae \\
Porphyromonadaceae & Ruminococcaceae \\
Rikenellaceae & Veillonellaceae \\
Enterococcaceae & Erysipelotrichaceae \\
Streptococcaceae & Alcaligenaceae \\
Turicibacteraceae & Enterobacteriaceae \\
Clostridiaceae & Other \\
\hline
\end{tabular}

Families observed b

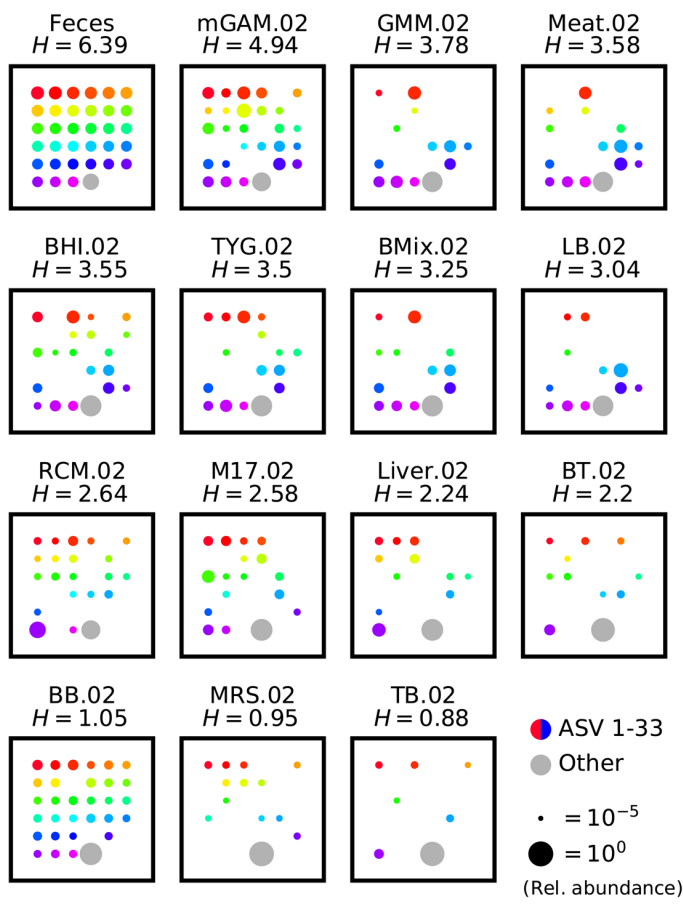

C

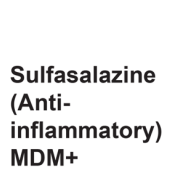

d

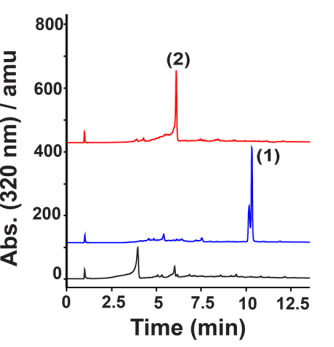<smiles>CC(C(=O)O)c1cccc(C(=O)c2ccccc2)c1</smiles>

Ketoprofen

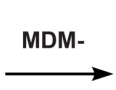<smiles>CC(C(=O)O)c1cccc(C(=O)c2ccccc2)c1</smiles>

(3)

Ketoprofen

Parent drug

MDM-Screen

HD-1

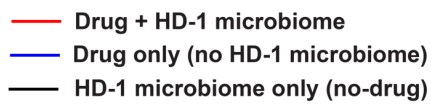


Figure 2. Development of MDM-Screen. a) Family level bacterial composition

215 of the original HD-1 fecal sample (far left), as well as that of HD-1 ex vivo cultures

216 grown anaerobically in 14 different media over two days (.01 and .02). Full names of the

217 media used are listed in the Methods. A four-day time course of HD-1 in the same

218 media is shown in Supplementary Fig. 1. 16S rRNA gene sequences that could not be

219 classified at the family level, and families with less than $1 \%$ relative abundance in all

220 samples are grouped into "Other". Cultures are ordered according to their Jensen-

221 Shannon DJs divergence from the original HD-1 sample (upper axes, computed at the

222 family level), where lower values indicate higher similarity to HD-1. Note that cultures

223 grown in mGAM (mGAM.02 and mGAM.01) are the most similar to HD-1. b) Amplicon

224 Sequence Variant (ASV) level bacterial composition of the original HD-1 fecal sample,

225 and that of day two ex vivo cultures of HD-1 grown in 14 different media, where each

226 square represents one sample. Rainbow colored dots represent the relative abundance

227 of individual ASVs that are above 1\% in HD-1, while grey dots represent the combined

228 relative abundance of all ASVs below $1 \%$ in HD-1. A larger dot indicates a higher

229 relative abundance, as indicated by a size scale at the bottom right corner. Samples are

230 ordered by their Shannon diversity $(H)$ at the ASV level, computed in bits and shown

231 above each square. Note that mGAM.02 culture has the highest Shannon diversity, and

232 the closest to HD-1. c) HPLC-MS analysis of sulfasalazine (1) incubated with HD-1

233 mGAM-02 culture (red) or with mGAM.02 broth (blue). A similar analysis is also done for

234 HD-1 mGAM.02 culture with no drug added (black). An HPLC chromatogram at an

235 absorbance of $320 \mathrm{~nm}$ is shown for all three samples, indicating the conversion of

236 sulfasalazine (1) to sulfapyridine (2) in the presence of the HD-1 microbiome. This is a 
237 typical case of an MDM+ drug. d) A similar HPLC-MS analysis for ketoprofen (3). An

238 HPLC chromatogram at an absorbance of $250 \mathrm{~nm}$ is shown, indicating no modification

239 to the parent drug in the presence of the HD-1 microbiome. This is a typical case of an

240 MDM- drug.

241

242

243

244

245

246

247

248

250

251

252

253

254

255

256

257

258

259 


\section{A high-throughput drug screen for MDM}

261

With an optimized ex vivo culturing system in hand, we developed the second arm of MDM-Screen: a combined biochemical / analytical chemistry approach for the systematic mapping of MDM. Our approach needed to fulfill the following criteria: a) is reproducible, and its reproducibility can be quickly assessed, b) is scalable to hundreds of drugs, c) is sensitive, even with a small amount of drug, and d) is feasible in a reasonable time frame and in an academic laboratory setting. After several iterations,

267 we successfully devised a strategy that meets all four desired criteria (Fig.1 and Fig. 2b, 2c). In this strategy, three samples are prepared per drug of interest: 1) a 3-ml, 24hour mGAM ex vivo culture of the starting human feces, incubated with the drug of

270 interest at a final concentration of $33 \mu \mathrm{M}$ (which is in line with estimates of drug

271 concentrations in the gastrointestinal tract) ${ }^{23}, 2$ ) a similar culture incubated with the

272 same volume of a vehicle control (DMSO), and 3) a 3-ml volume of sterile mGAM,

273 incubated with the same drug concentration. The no-drug control is important to

274 distinguish microbiome-derived small molecules from ones that result from MDM of the

275 tested drug. The no-microbiome control is important to distinguish cases of passive drug

276 degradation or faulty chemical extraction from those of active MDM. Cultures and

277 controls are then incubated for an additional 24 hours at $37^{\circ} \mathrm{C}$ in an anaerobic chamber,

278 chemically extracted, and finally analyzed using High Performance Liquid

279 Chromatography coupled with Mass Spectrometry (HPLC-MS). The entire procedure is 280 repeated three consecutive times to verify the reproducibly of the screen. 
283 diverse in structure and biological activities (erythromycin, antibiotic; terbinafine,

284 antifugal; ketoprofen, antiinflammatory; valganciclovir, antiviral; topotecan, anticancer;

285 atenolol, antihypertensive). Importantly, we also included a drug that is known to be

286 readily metabolized by the human microbiome as a positive control: sulfasalazine, a

287 prodrug that is intestinally activated by the human microbiome to produce the anti-

288 inflammatory drug 5-aminosalicylic acid (5-ASA) and the metabolite sulfapyridine ${ }^{24,25}$.

289 Unequivocally, we observed a reproducible metabolism of sulfasalazine into

290 sulfapyridine, while the rest of the tested drugs remained unchanged in all three trials

291 (Fig. 2c, 2d). These results establish our analytical screen as a valid method for

292 determining the effect of MDM on orally administered drugs, where positive and

293 negative results can be readily and reproducibly differentiated.

With these promising results from the pilot assay, we decided to apply MDM-

295 Screen to a library of 575 orally administered drugs. This library is a subset of the

296 SCREEN-WELL ${ }^{\circledR}$ FDA approved drug library (Enzo Life Sciences, Inc.), including only

297 drugs with an established oral route of administration. We chose this library because of

298 its diversity in chemical structure and pharmacological activity (Supplementary Table

299 1); and although all of the drugs in this library are currently being used in the clinic,

300 almost nothing is known about their metabolism by the human gut microbiome.

301 Following the procedures established in the pilot screen, we tested each drug twice,

302 along with matching no-drug and no-microbiome controls. For final verification and

303 consensus determination, a third trial was performed for drugs that showed a positive

304 MDM on either or both of the first two trials. Therefore, a drug is deemed MDM+ when it

305 is metabolized in the same manner during at least two out of three independent 
experiments. Taken together, we have developed and performed a high-throughput screen for mapping the ability of the complex human microbiome to metabolize orally administered small molecule drugs, in a systematic and unbiased manner.

MDM-Screen identifies novel drug-microbiome interactions

Among the 575 drugs tested, 438 (76\%) of them were successfully analyzed

issues related to drug stability or incompatibilities with the extraction or chromatography methods employed (see Discussion). Among the successfully analyzed drugs, 57 (13\%) were identified as MDM-Positive (MDM+) (Supplementary Table 1,

316 Supplementary Table 2, and Supplementary Fig. 3). As expected, several previously

317 reported MDM cases were identified, further verifying MDM-Screen as a systematic

318 method for discovering microbiome-drug interactions. These include the nitroreduction

319 of the muscle relaxant dantrolene ${ }^{26}$, nitroreduction of the antiepileptic clonazepam

320 (reported only in rats before this study $)^{27}$, hydrolysis of the isoxazole moiety in the

321 antipsychotic risperidone ${ }^{28,29}$, as well as several modifications to the bile acids

322 chenodeoxycholic acid and ursodiol ${ }^{30}$.

More importantly, MDM-Screen identified a suite of novel MDM cases (46 cases,

$32480 \%$ of the MDM+ drugs). Among those, we selected four examples for detailed

325 characterization: the commonly used anti-hypertensive / cardiac drug nicardipine, the

326 chemotherapeutic agent capecitabine, and finally, the two steroidal anti-inflammatory

327 drugs hydrocortisone (cortisol) and hydrocortisone acetate (often administered rectally),

328 which produce an identical MDM metabolite. To unequivocally determine the structure 
329 of the resulting metabolite for each of these cases, we scaled up the biochemical

330 incubation with HD-1, isolated and purified each of the resulting metabolites, and

331 elucidated their structures using Nuclear Magnetic Resonance (NMR) (see Methods

332 and Supplementary Data 1). Nicardipine metabolite (aminonicardipine) corresponds to

333 the nitroreduced form of the drug: a common modification by members of the gut

334 microbiome but one that has not been reported for this drug (Fig. 3a). For

335 hydrocortisone, we determined that MDM results in the reduction of the ketone group at

336 C20, producing 20-dihydrocortisone (Fig. 3b). For hydrocortisone acetate, the same

337 modification occurs but is accompanied with deacetylation of the C21 hydroxyl group

338 (Supplementary Fig. 3). While C20 reduction was previously reported for

339 hydrocortisone ${ }^{31,32}$, neither deacetylation nor C20 reduction were reported for

340 hydrocortisone acetate. For capecitabine, we show that MDM results in complete

341 deglycosylation, again, a modification never reported for this drug (Fig. 3c). Taken

342 together, these results establish MDM-Screen as a viable method for identifying both

343 known and novel biochemical modifications of structurally and pharmacologically

344 diverse drugs by the gut microbiome. 
bioRxiv preprint doi: https://doi.org/10.1101/538215; this version posted February 3, 2019. The copyright holder for this preprint (which was not certified by peer review) is the author/funder, who has granted bioRxiv a license to display the preprint in perpetuity. It is made available under aCC-BY-NC-ND 4.0 International license.

352

a

Nicardipine

(Anti-

hypertensive)

MDM+

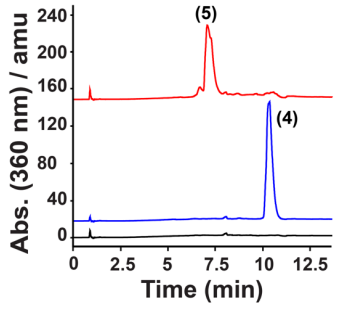

b

Hydrocortisone (Steroidal

anti-

inflammatory)

MDM+

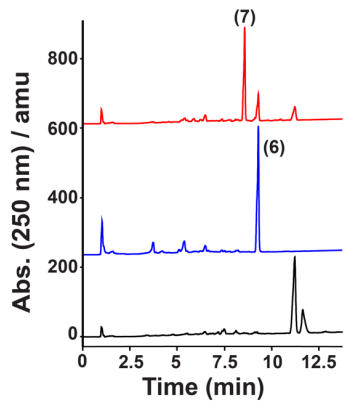

\section{C \\ Capecitabine (Anticancer) MDM+}

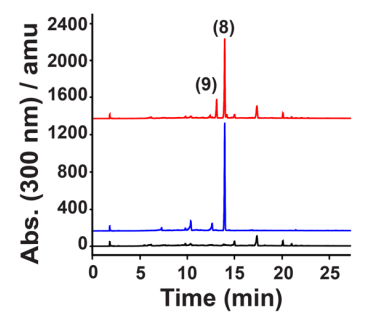

Drug + HD-1 microbiome Drug only (no HD-1 microbiome) HD-1 microbiome only (no-drug)

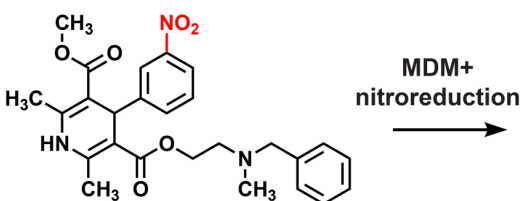

Nicardipine

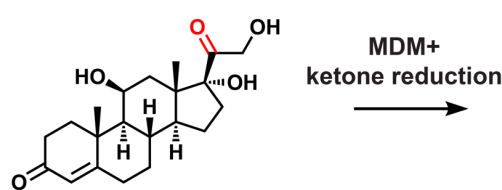

(6)

Hydrocortisone

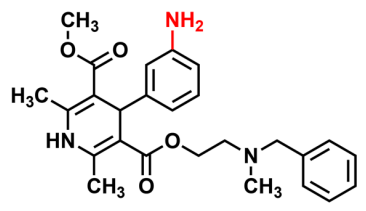

(5)

Aminonicardipine

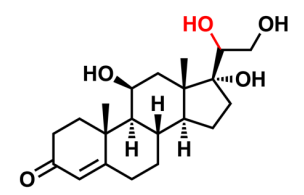

(7)

20-dihydrocortisone

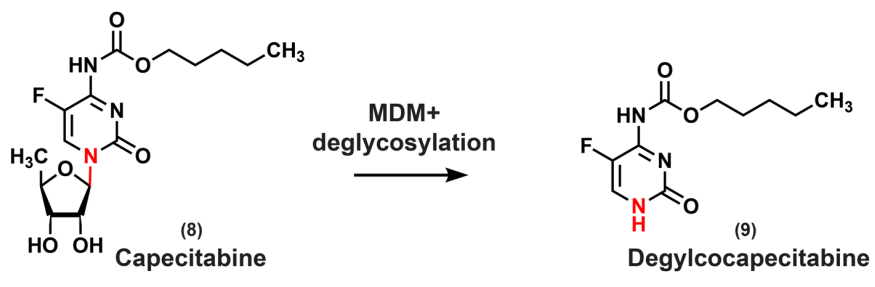

Parent drug HD-1

Resulting metabolite 
Figure 3. Examples of positive hits from MDM-Screen. An HPLC-MS analysis

364 is shown for each of the selected examples, where three chromatograms are displayed

365 per case: one for the drug incubated with HD-1 mGAM.02 culture (red), a second one

366 for the drug incubated with mGAM.02 broth (blue), and a third one for HD-1 mGAM.02

367 culture with no drug added (black). a) An HPLC chromatogram at an absorbance of 360

$368 \mathrm{~nm}$ is shown for nicardipine (4), indicating its conversion to aminonicardipine (5) in the

369 presence of the HD-1 microbiome. b) An HPLC chromatogram at an absorbance of 250

$370 \mathrm{~nm}$ is shown for hydrocortisone (6), indicating its conversion to 20-dihydrocortisone (7)

371 in the presence of the HD-1 microbiome. c) An HPLC chromatogram at an absorbance

372 of $300 \mathrm{~nm}$ is shown for capecitabine (8), indicating its conversion to

373 deglycocapecitabine (9) in the presence of the HD-1 microbiome. Structures of the three

374 metabolites were elucidated using NMR (see Supplementary Data 1).

375

376

377

378

379

380

381

382

383

384

385 


\section{A global analysis of MDM by HD-1}

Other than discovering novel drug-microbiome interactions, the results of our

systematic screen allow for an unbiased, global analysis of MDM. Overall, the $57 \mathrm{MDM}+$ drugs belonged to 28 pharmacological classes and an even more diverse set of

members of the microbiome would be more likely to metabolize natural or naturally-

categories: naturally occurring molecules (i.e., molecules directly derived from humans, naturally occurring molecules (i.e., a semisynthetic derivative or a close structural mimic

397 of a natural product, an example of this category is hydrocortisone acetate; $N=90$ ), and synthetic molecules (an example of this category is nicardipine; $\mathrm{N}=318$ ). Interestingly, derivative, 26 out of $120,21.6 \%$ ) to that of the third category (synthetic, 31 out of 318 , $40110 \%)$, we revealed a significant difference $(p<0.001$, two-tailed proportions $z$-test).

402 Intrigued, we decided to examine differences in MDM at lower levels of drug 403 classification. We observed a significantly higher hit rate among steroids (steroids: 14 404 out of $26,53.8 \%$; non-steroid: 43 out of $412,10.4 \%, p<0.001$, two-tailed proportions z405 test), including hormonal steroids, corticosteroids, bile acids, and derivatives thereof. In 406 fact, the high hit rate of the steroid class is the major contributor to the observed

407 difference between the hit rates of natural/derivative and synthetic groups, which is 408 abolished upon exclusion of the steroids (non-steroid natural/derivative: 12 out of 96 , 
$12.5 \%$; non-steroid synthetic: 31 out of $316,10 \%)$. The high hit rate among steroids is

410 in-line with the idea that the microbiome is more likely to metabolize compounds it

411 frequently encounters, as steroids (e.g., bile acids) are normally present in the gut, and

412 at high concentrations ${ }^{33}$. The fact that $\sim 10 \%$ of fully synthetic molecules are

413 metabolized by HD-1 indicates the presence of a yet-unexplored range of biochemical

414 activities that are encoded by the gut microbiome, and are capable of recognizing

415 foreign substrates.

Linking MDM to specific members of the human microbiome

Our results from MDM-Screen indicate a significant and diverse ability of the

419 collective gut microbiome to metabolize clinically used drugs that are unrelated in

420 structure and biological activity. Next, we wondered whether the observed biochemical

421 modifications can be attributed to specific members of the microbiome. To answer this

422 question, we picked the same representative set of MDM transformations that we

423 characterized above (3 transformations on 3 drugs) (Fig. 3), and explored the ability of

424 a limited panel of 11 gut microbiome isolates and a laboratory strain to perform them.

425 This panel was selected from three of the most abundant Phyla that normally inhabit the 426 gut microbiome (Firmicutes, Bacteroidetes, and Proteobacteria), and spans 10 bacterial

427 genera. Overall, nitroreduction of nicardipine was extensively performed by

428 Bacteroidetes and Firmicutes, while capecitabine deglycosylation was mainly performed 429 by Proteobacteria and one of the two tested Bacteroidetes: Parabacteroides distasonis.

430 (Fig. 4b). None of the tested strains performed C20 reduction of hydrocortisone,

431 suggesting that it is performed by a yet unidentified member(s) of the HD-1 microbiome 
432 (only two gut isolates were previously shown to perform C20 reduction on

433 hydrocortisone: Clostridium scindens and Butyricicoccus desmolans) ${ }^{31,32}$.

$434 \quad$ Interestingly, we also observed sequential MDM transformations that appear to

435 be contributed by different members of the microbiome on the same parent drug. An

436 example of this includes the deacetylation (ester hydrolysis) and further reduction of

437 hydrocortisone acetate. When hydrocortisone acetate (10) is incubated with either $P$.

438 distasonis or Clostridium bolteae, it is deacetylated to yield hydrocortisone. When

439 incubated with HD-1, however, it is both deacetylated and further reduced to yield 20-

440 dihydrocortisone (Fig. 4c and Supplementary Fig. 4). Since we determined that a yet-

441 unidentified member of the HD-1 microbiome is able to reduce hydrocortisone (6) at

442 C20 (Fig. 3 and Fig. 4b), a two-step metabolic sequence is likely at play here, where

443 hydrocortisone acetate (10) is first deacetylated to yield hydrocortisone (6) by

444 Parabcteroides or Clostridium sp. in HD-1, then ketone reduced at C20 by another

445 member of the microbiome to yield 20-dihydrocortisone (7). Overall, these results

446 highlight the utility of our approach in mapping the ability of the complex human

447 microbiome to metabolize drugs, whether it is contributed by one or several members of

448 the microbiome: a key advance over experiments that are based on a single isolate. 
bioRxiv preprint doi: https://doi.org/10.1101/538215; this version posted February 3, 2019. The copyright holder for this preprint (which was not certified by peer review) is the author/funder, who has granted bioRxiv a license to display the preprint in perpetuity. It is made available under aCC-BY-NC-ND 4.0 International license.

a

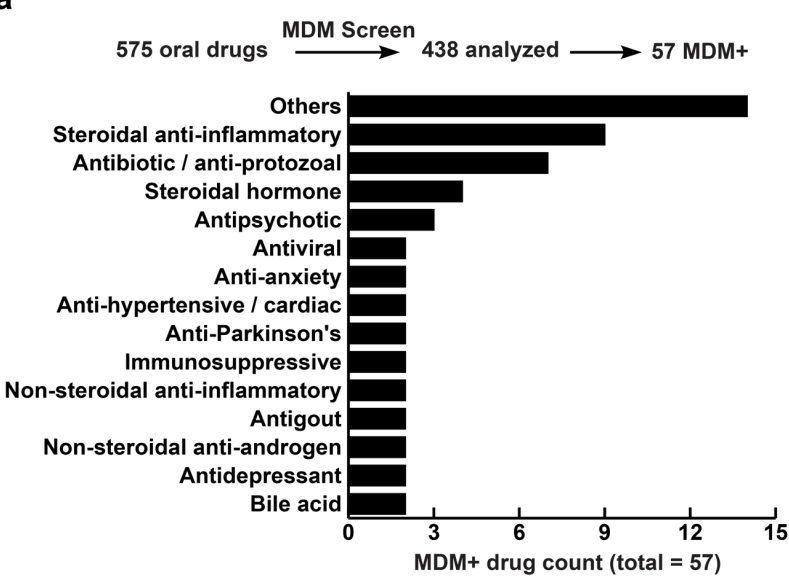

b

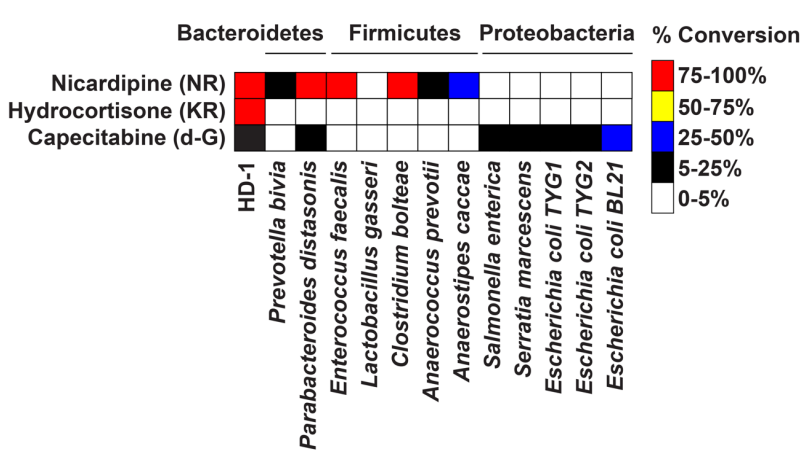

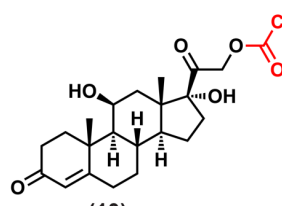

(10)

Hydrocortisone

acetate

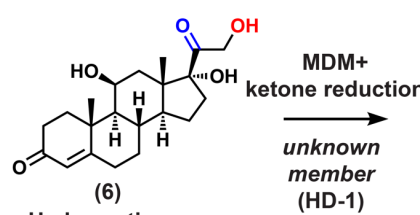

Hydrocortisone

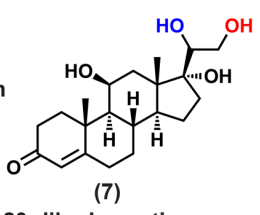

20-dihydrocortisone 
Figure 4. Overall results of MDM-Screen. a) A bar graph showing the pharmacological classes of MDM+ drugs discovered by MDM-Screen. "Others" include

471 one drug each from 14 additional classes (Supplementary Table 1). In the inset bar

472 graph, "natural", "derivative", or "synthetic" indicate whether the tested drug is: a natural

473 product of any source (human, plant, microbial), a derivative of a naturally occurring

474 molecule, or fully synthetic, respectively. ${ }^{* * *}$ indicates $p<0.001$, two-tailed proportions

475 z-test. b) A heat map indicating the ability of each of 12 tested strains to perform the

476 three example modifications in Fig. 3: NR, nitroreduction; KR, ketone reduction; and d-

477 G, deglycosylation. c) An example of sequential metabolism revealed by MDM-Screen:

478 hydrocortisone acetate (10) can be first deacetylated by two members of the

479 microbiome ( $P$. distasonis and $C$. bolteae) to yield hydrocortisone (6), which is then

480 reduced at C20 by a yet-unidentified member of the HD-1 microbiome to yield 20-

481 dihydrocortisone (7). Structures were confirmed by NMR and comparison to authentic

482 standards (Supplementary Data 1 and Supplementary Fig. 4). 


\section{An MDM case study: capecitabine}

Our ability to map MDM in a systematic manner is only the first step towards

494 understanding the mechanistic details and biological consequences of direct drug-

495 microbiome interactions. Therefore, we selected one MDM example, deglycosylation of

496 capecitabine, for follow-up studies. Five main reasons motivated us to choose this

497 modification for additional studies. First, the modification exerted on capecitabine yields

498 a novel metabolite (deglycocapecitabine) that has not been previously reported in

499 humans or animals, potentially providing more insights into the complex

500 pharmacokinetics of this drug. Second, capecitabine is one of several generations of

501 antimetabolite chemotherapeutic agents, many of which are prodrugs for 5-fluorouracil

502 (5-FU), and are known collectively as the oral fluoropyrimidines (FPs) ${ }^{34,35}$. Because

503 these agents share the same overall structure (a glycosylated and fluorinated

504 pyrimidine), they may be subject to the same MDM. Third, oral FPs' bioavailability and

505 toxicity vary widely among patients ${ }^{36,37}$, but the human gut microbiome's contribution to

506 this variability has not been explored. Fourth, a related transformation was previously

507 reported for another pyrimidine analog, the antiviral sorivudine, and linked to toxic

508 outcomes during co-administration with 5-FU, suggesting the potential yet unexplored

509 importance of deglycosylation for a wide range of drugs ${ }^{38}$. Finally, as shown above,

510 capecitabine MDM is performed mainly by proteobacterial members of the microbiome,

511 as well as some members of the Bacteroidetes. This feature not only provides

512 genetically tractable organisms for functional studies (e.g., E. coli), but may also result

513 in MDM variability between individuals depending on the relative abundance of specific

514 metabolizers. 


\section{Genetic basis of MDM deglycosylation}

517 sought to identify microbiome-derived enzymes responsible for this transformation. In

518 humans, thymidine phosphorylase (TP) and uridine phosphorylase (UP), both part of

519 the pyrimidine salvage pathway, were shown to catalyze the required deglycosylation of

520 5'-deoxy-5-fluorouridine at the last step of capecitabine metabolism to yield 5-FU ${ }^{39}$. To

521 test whether bacterial homologs of human TP and/or UP are responsible for the

522 observed MDM deglycosylation of capecitabine, we generated strains of E. coli

523 BW25113 that are knockouts for TP $(\Delta d e o A)$, UP $(\Delta u d p)$, or both, and compared their

524 ability to metabolize capectitabine to that of wild type E. coli (Fig. 5a). While wild type E.

525 coli efficiently deglycosylates capecitabine ( $30 \%$ conversion rate), the deglycosylating

526 activity of $\Delta u d p$ and the $\Delta d e o A / \Delta u d p$ knockout strains is significantly diminished (less

527 than 4\% conversion rate, $p$-value $<0.001$, two-tailed t-test) (Fig. 5b). Surprisingly, the

$528 \triangle d e o A$ knockout strain showed a significant increase in its deglycosylating activity in

529 comparison to the wild type ( 50\% conversion rate, $p$-value $<0.01$, two-tailed t-test),

530 possibly due to a compensating mechanism (e.g., overexpression of $u d p$ ) in the

531 absence of $d e o A$. These results indicate that microbiome-derived UP is, at least in part,

532 responsible for the intestinal deglycosylation of capecitabine.

534 MDM deglycosylation is widespread in the fluoropyrimidine class of

\section{chemotherapeutic agents}

Next, we wondered whether deglycosylation occurs with other FPs, and whether

537 the same enzymes are involved. To answer this question, we investigated the MDM of 
538 two additional oral FPs (doxifluridine and trifluridine), using both WT and mutant E. coli.

539 We found that both drugs were subject to the same MDM deglycosylation, indicating

540 that this modification is widespread among this class of molecules. Interestingly, unlike

541 with capecitabine, almost complete deglycosylation was observed with WT E. coli (there

542 was hardly any parent molecule left after 24 hours), and the activity was dependent on

543 both TP and UP, as it was abolished only in the $\Delta d e o A / \Delta u d p$ knockout (Supplementary

544 Fig. 5 and Supplementary Fig. 6). These results indicate a level of deglycosylation

545 specificity for TP/UP amongst the FPs, likely due to how well each drug mimics their

546 natural substrate. Remarkably, the consequences of the same modification may be very

547 different depending on the structural features of the tested drug. In the case of

548 trifluridine, the resulting metabolite (trifluorothymine) is inactive (Fig. $\mathbf{5 d}$ and

549 Supplementary Fig. 5): trifluridine needs to be incorporated intact into DNA to cause

550 cytotoxicity ${ }^{40}$. Such a premature intestinal inactivation by the microbiome may thus be

551 an unknown contributor to the established low bioavailability of trifluridine, in addition to

552 the known contribution of human $\mathrm{TP}^{36}$. In the case of doxifluridine, however, the

553 resulting metabolite is the active 5-FU itself (Fig. 5e and Supplementary Fig. 6). This

554 premature activation of the prodrug may therefore lead into gastrointestinal toxicity -

555 again, a side effect commonly associated with oral doxifluridine ${ }^{41,42}$.

556 To shed light on the potential consequences of capecitabine MDM

557 deglycosylation, we sought to interrogate whether its metabolite, deglycocapecitabine,

558 is able to re-enter the normal capecitabine metabolism cycle and yield 5-FU. In the liver,

559 capecitabine is metabolized by liver carboxyesterases to yield 5'-deoxy-5-fluorocytidine,

560 which is then deaminated by cytidine deaminase to yield 5'-deoxy-5-fluorouridine 
561 (doxifluridine). Preferentially in tumor tissues (due to the higher expression level of its

562 metabolizing enzymes), doxifluridine is deglycosylated by human TP/UP to yield the

563 active 5-FU (Supplementary Fig. 7) ${ }^{43}$. Similarly, deglycocapecitabine would almost

564 certainly need to be processed by liver carboxyesterases to yield 5-fluorocytidine. Thus,

565 we decided to directly test the activity of human carboxyesterase 1 (CES1) - the most

566 important caroboxyesterase in capecitabine metabolism - against

567 deglycocapecitabine ${ }^{44,45}$. Notably, while CES1 efficiently removed the carbamate group

568 from capecitabine to yield 5'-deoxy-5-fluorocytidine in vitro, deglycocapecitabine was

569 not recognized as a substrate by the enzyme under the same conditions

570 (Supplementary Fig. 7). These results suggest that capecitabine MDM deglycosylation

571 results in an inactivated product that is unlikely to yield the active 5-FU. Taken together,

572 our findings indicate that FP deglycosylation is a common yet understudied MDM

573 transformation that may have diverse consequences on the pharmacokinetics and/or

574 pharmacodynamics of this widely used class of chemotherapeutic agents.

575

576

577

578

579

580

581

582

583 
bioRxiv preprint doi: https://doi.org/10.1101/538215; this version posted February 3, 2019. The copyright holder for this preprint (which was not certified by peer review) is the author/funder, who has granted bioRxiv a license to display the preprint in perpetuity. It is made available under aCC-BY-NC-ND 4.0 International license.

a

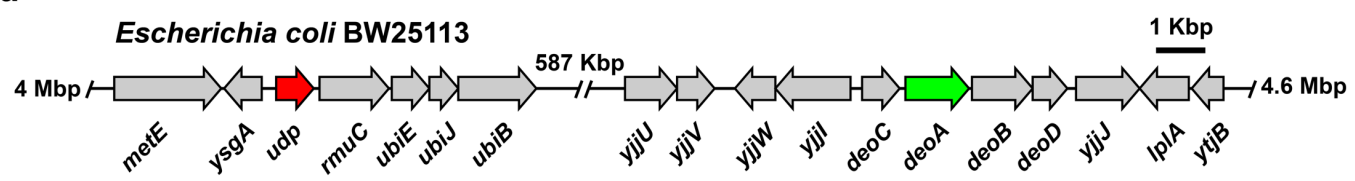

b

C
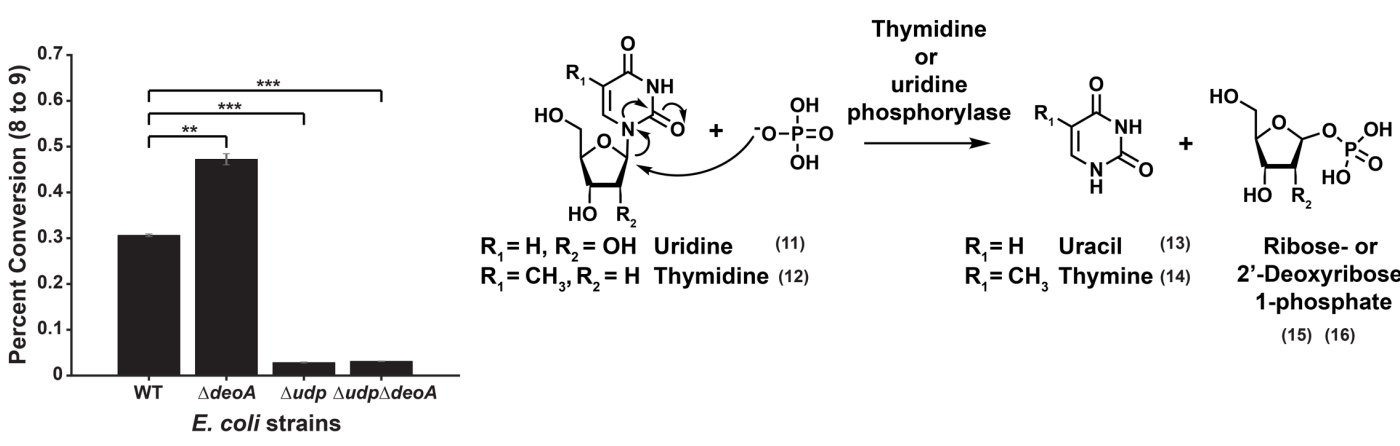

$\mathbf{R}_{1}=\mathrm{H}, \mathbf{R}_{2}=\mathrm{OH}$ Uridine (11)

$\mathrm{R}_{1}=\mathrm{H} \quad$ Uracil (13) Ribose- or

$\mathbf{R}_{1}=\mathrm{CH}_{3}, \mathbf{R}_{2}=\mathrm{H}$ Thymidine (12)

$\mathbf{R}_{1}=\mathrm{CH}_{3}$ Thymine $(14)$

2'-Deoxyribose-

1-phosphate

(15) (16)

d

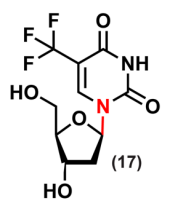

Trifluridine

(anticancer)

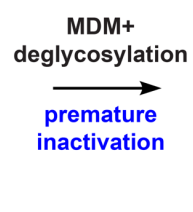

584

585

586

587

588

589

590

591

592

593

594

595 e<smiles>O=c1[nH]cc(C(F)(F)F)c(=O)[nH]1</smiles>

Trifluorothymine (inactive)

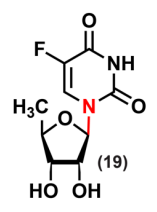

Doxifluridine (anticancer)
MDM+ deglycosylation $\overrightarrow{\text { premature }}$ activation

5-Fluorouracil (active)

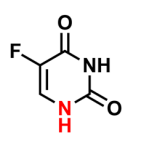

(20) 
Figure 5. Genetic basis and widespread nature of MDM deglycosylation

597 among the FPs. a) Genetic organization of the $u d p$ and $d e o A$ loci in the genome of $E$.

598 coli BW25113. b) A bar graph indicating percent conversion of capecitabine (8) to

599 deglycocapecitabine (9) by wild type E. coli BW25113 (WT), and $\Delta u d p, \Delta d e o A$, and

$600 \Delta d e o A / \Delta u d p$ mutants (each tested in triplicate). ${ }^{* * *}$ indicates $p$-value $<0.001$, while ${ }^{* *}$

601 indicates $p$-value $<0.01$, two-tailed t-test. Error bars represent the standard deviation. c)

602 Biochemical reaction catalyzed by thymidine and uridine phosphorylases on their

603 natural substrates. d) MDM deglycosylation of the oral anticancer drug trifluridine (17)

604 leads to its premature inactivation, since trifluorothymine (18) is no longer active. e)

605 MDM deglycosylation of the anticancer prodrug doxifluridine (19) leads to its premature

606 activation, since 5-fluorouracil (20) is the intended active metabolite. MDM

607 deglycosylation of trifluridine and doxifluridine is also dependent on $d e o A$ and $u d p$, and

608 the structures of all resulting metabolites were confirmed by comparison to authentic

609 standards (Supplementary Fig. 5 and Supplementary Fig. 6). 


\section{MDM deglycosylation occurs in vivo}

620

621 including MDM deglycosylation of FPs, it is unclear whether these results (observed ex

622 vivo) can be recapitulated within a host (in vivo). To address this question, we selected

623 MDM deglycosylation of capecitabine as a proxy for other FPs, and monitored it in an in

624 vivo pharmacokinetic study that is performed in a microbiome-dependent manner. We

625 treated two groups of C57/B6 mice with a cocktail of antibiotics for 14 days, then

626 colonized one group with HD-1 while the control group remained non-colonized. The

627 two groups were then treated with a single human-equivalent oral dose of capecitabine

628 (755 mg/kg), and blood and feces were collected from each mouse at times 0, 20, 40,

62960,120 and 240 minutes post drug administration (Fig. 6a). Finally, we quantified

630 capecitabine and its metabolites in chemical extracts from blood and feces using HR-

631 HPLC-MS. Remarkably, deglycocapecitabine was detected in fecal samples from

632 animals colonized with HD-1 as early as 20 min after dosing, and was almost

633 completely absent in non-colonized ones (Fig. 6b). To our surprise, with the single dose

634 regimen provided here, we could not detect deglycocapecitabine in mouse blood

635 samples. In contrast, capecitabine, and its major liver-derived metabolite (5'-deoxy-5-

636 fluorocytidine) were readily detected in blood with no significant differences between the

637 two groups (Supplementary Fig. 8). These results indicate that - at least in the case of

638 FP deglycosylation - MDM transformations observed ex vivo by MDM-Screen are recapitulated in vivo. 
a

Colonize with HD-1 (Group 1) Keep non-colonized (Group 2)

$\downarrow \downarrow$

Days

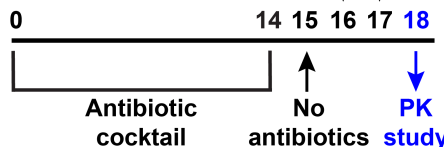

cocktail

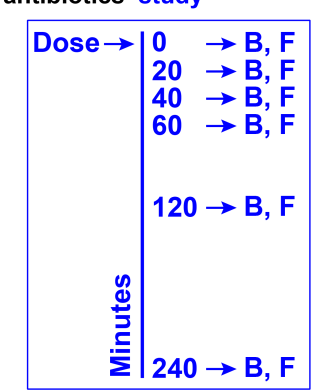

b

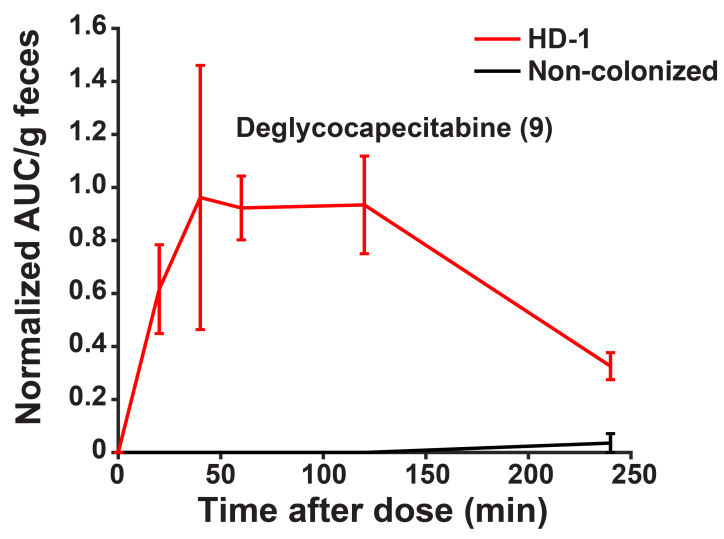

Figure 6. MDM deglycosylation occurs in vivo. a) Design of a microbiome-

645 dependent pharmacokinetic experiment performed in mice using capecitabine. Mice are treated with antibiotics for 14 days, then colonized with HD-1 $(\mathrm{N}=6)$ or left non-colonized

$647(\mathrm{~N}=6)$. On the pharmacokinetic experiment day, a single human-equivalent dose is

648 administered to mice using oral gavage, and serial sampling of blood $(B)$ and feces $(F)$

649 is performed at $0,20,40,60,120$, and 240 minutes post dosing. b) HR-HPLC-MS

650 based quantification of deglycocapecitabine in fecal samples from mice colonized with

651 HD-1 in comparison to non-colonized ones (see also Supplementary Fig. 8).

652 Metabolite Area Under the Curve (AUC) per gram of feces is normalized by the AUC of

653 an internal standard (voriconazole) (see Methods). Error bars represent the standard

654 error of the mean.

655

656 


\section{DISCUSSION}

In the current study, we develop a systematic screen for assessing the ability of

662 the human gut microbiome to directly metabolize orally administered drugs, using a

663 combination of microbial community cultivation, a high-throughput drug screen, bacterial

664 genetics, and defined mouse colonization assays. Several key differences set our

665 approach apart from previous studies in this area. First, instead of relying on single

666 isolates in performing the initial screen, we use a well-characterized patient-derived

667 microbial community that mimics to a large extent the original sample in composition

668 and diversity. Despite the technical challenges associated with characterizing and

669 maintaining stable microbial communities in batch cultures, three main advantages

670 make this strategy worth pursuing: i) the extent of a biochemical transformation

671 performed by single isolates cultured individually may be completely different than that

672 performed by the same isolates when cultured as part of a complex community; ii) the

673 net result of several members of the microbiome acting on the same drug can only be

674 identified in mixed communities and not in single-isolate experiments, unless all

675 pairwise and higher order permutations are tested; and iii) our strategy is

676 "personalizable". Some of the results obtained here - including the extent and type of

677 certain modifications - will likely be specific to the strain-level composition of the HD-1

678 microbiome, and may vary if the assay is repeated with samples from different subjects.

679 MDM-Screen has thus a good potential for assessing inter-patient variability in MDM.

680 Second, while most previous studies have focused on certain drug / species

681 combinations that have historically been deemed important, our screen is agnostic 
682 towards the modifications being detected, the drugs being screened, and the

683 responsible members of the microbiome being identified. This unbiased, systematic

684 approach allowed us to map for the first time the potential extent of MDM, and to

685 discover drug-microbiome interactions never reported before. We provide these results

686 as a resource for the scientific community to further study the mechanistic details and

687 pharmacological consequences of these newly discovered interactions. Third, MDM-

688 Screen is performed in an efficient, high-throughput manner for both the organisms (a

689 complex microbial community mimicking the original microbiome sample), and the drugs

690 tested (almost 600 drugs were tested in an academic lab setting). With additional

691 optimizations on the cultivation side (e.g., the use of 96-well plates) and the analytical

692 chemistry side (e.g., automation of the extraction procedures), one can easily expand

693 the screen to hundreds of human microbiome samples and thousands of drugs.

Despite these advances, our approach is still subject to several limitations. First,

$24 \%$ of the drugs tested failed to be analyzed using the general analytical chemistry

workflow described in the initial MDM-Screen. These drugs fell into one or more of three

697 main categories: were not stable after overnight incubation in no-microbiome controls,

698 could not be extracted using ethyl acetate, or could not be analyzed using reverse

699 phase chromatography, with the last two being attributed mostly to polar or charged

700 compounds. An alternative chemical analysis method will need to be developed for

701 these molecules in order to assess their MDM. Second, we focused initially on oral

702 drugs, yet several parenteral drugs and their liver-derived metabolites may be subject to

703 important MDM transformations after biliary secretion. Third, even in our most diverse

704 ex vivo cultures, we fail to support the growth of $100 \%$ of the community in the original 
sample. Finally, we initially based our analysis on a single human sample, HD-1.

706 Therefore, it is almost certain that the types of MDM transformations observed here are

707 an underestimation of all possible ones, and that performing MDM-Screen several times

708 with samples derived from unrelated subjects may be necessary to reveal the complete

709 biochemical potential of MDM.

710

Although MDM was shown to lead into changes in the bioavailability, toxicity,

711 and/or efficacy of certain therapeutics (e.g., digoxin) - to the same extent as liver

712 metabolism - it is almost entirely overlooked by the regulatory agencies when

713 developing new drugs ${ }^{14,46}$. Our current study was designed to achieve two main goals:

714 a) develop a simple platform for studying MDM in a systematic manner; b) map the

715 extent of MDM against commonly used drugs, including the functional characterization

716 of key proof-of-principle examples. By achieving these goals, our overall findings reveal

717 an unexpectedly large and diverse ability of the human microbiome to directly

718 metabolize clinically used, small molecule drugs, and a wide potential for MDM as a key

719 factor in explaining the observed inter-patient variability in the pharmacokinetics and/or

720 pharmacodynamics of these agents. At the same time, our approach provides the

721 regulatory agencies (e.g., the Food and Drug Administration) with a simple screen for

722 assessing MDM that can be easily implemented in any typical drug development

723 pipeline. It is crucial that drug-microbiome interactions, including both effects of drugs

724 on the microbiome (which were systematically mapped in an elegant screen published

725 recently) ${ }^{23}$, as well as MDM (mapped here for the first time) are considered while

726 studying the pharmacology and toxicology of newly developed therapeutic agents. 
METHODS

ex vivo culture of human gut microbiome communities

733 activity was not human subjects research. Consequently, Princeton IRB approval was

734 not applicable. Freshly collected human fecal material from a healthy donor, HD-1 ( 30

735 min from collection, transported on ice) was brought into an anaerobic chamber (70\%

$736 \mathrm{~N}_{2}, 25 \% \mathrm{CO}_{2}, 5 \% \mathrm{H}_{2}$ ). One gram of the sample was suspended in $15 \mathrm{ml}$ of sterile

737 phosphate buffer (PBSc) supplemented with $0.1 \%$ L-cysteine in a $50 \mathrm{ml}$ sterile falcon

738 tube. The suspension was left standing still for 5 min to let insoluble particles settle. The

739 supernatant was mixed with an equal volume of $40 \%$ glycerol in PBSc. Aliquots (1 ml) of

740 this suspension were placed in sterile cryogenic vials and frozen at $-80^{\circ} \mathrm{C}$ until use ${ }^{47}$.

742 of 14 different media: Liver Broth (Liver), Brewer Thioglycolate Medium (BT), Bryant and

743 Burkey Medium (BB), Cooked Meat Broth (Meat), Thioglycolate Broth (TB), Luria-

744 Bertani Broth (LB) (obtained from Sigma Aldrich, USA), Brain Heart Infusion (BHI), MRS

745 (MRS), Reinforced Clostridium Medium (RCM), M17 (M17) (obtained from Becton

746 Dickinson, USA), modified Gifu Anaerobic Medium (mGAM) (obtained HyServe,

747 Germany), Gut Microbiota Medium $\left(\mathrm{GMM}^{47}\right), \mathrm{TYG}$, and a 1:1 mix of each (BestMix), and

748 cultures were incubated at $37^{\circ} \mathrm{C}$ in an anaerobic chamber. One $\mathrm{ml}$ was harvested from

749 each culture each day for 4 consecutive days, and centrifuged to recover the resulting

750 bacterial pellets. DNA was extracted from all pellets using the Power Soil DNA Isolation 
751 kit (Mo Bio Laboratories, USA), the 16S rRNA gene was amplified ( 250 bps, V4

752 region), and Illumina sequencing libraries were prepared from the amplicons according

753 to a previously published protocol and primers ${ }^{48}$. Libraries were further pooled together

754 at equal molar ratios and sequenced on an Illumina HiSeq 2500 Rapid Flowcell as

755 paired-end (2X175 bps) reads, along with 8 bps Index reads, following the

756 manufacturer's protocol (Illumina, USA). Raw sequencing reads were filtered by

757 Illumina HiSeq Control Software to generate Pass-Filter reads for further analysis.

758 Different samples were de-multiplexed using the index reads. Amplicon sequencing

759 variants (ASVs) were then inferred from the unmerged pair-end sequences using the

760 DADA2 plugin within QIIME2 version 2018.6 $6^{16,17}$. The forward reads were trimmed at

$761165 \mathrm{bp}$ and the reverse reads were trimmed at $140 \mathrm{bp}$. All other settings within DADA2

762 were default. Taxonomy was assigned to the resulting ASVs with a naive Bayes

763 classifier trained on the Greengenes database version $13.8^{18,19}$. Only the target region

764 of the 16S rRNA gene was used to train the classifier. Rarefaction analysis was

765 performed within QIIME2 ${ }^{17}$.

766

767 ex vivo screening of the drug library

768 In an anaerobic chamber, a small $(\sim 100 \mu \mathrm{l})$ of an HD-1 glycerol stock was diluted

769 in $1 \mathrm{ml}$ of $\mathrm{mGAM}$, then $20 \mu \mathrm{l}$ of this solution was used to inoculate $3 \mathrm{ml}$ of $\mathrm{mGAM}$ in

770 culture tubes. Cultures were grown for 24 hours at $37^{\circ} \mathrm{C}$ in an anaerobic chamber. After

77124 hours, $10 \mu \mathrm{L}$ of each drug (the concentration of each molecule in the library is 10

$772 \mathrm{mM}$ ), or of a DMSO control were added to the growing microbial community. In addition,

$77310 \mu \mathrm{L}$ of each drug was also incubated similarly in a no-microbiome, mGAM control. 
774 HD-1 / DMSO control pellets from several batches of the screen were analyzed using

775 high-throughput 16S rRNA gene sequencing as described above to ensure the

776 maintenance of a similarly diverse microbial composition. Experiments and controls

777 were allowed to incubate under the same conditions for a second 24-hour period. After

778 incubation, cultures were extracted with double volume of ethyl acetate and the organic

779 phase was dried under vacuum using a rotary evaporator (Speed Vac). The dried

780 extracts were suspended in $250 \mu \mathrm{L} \mathrm{MeOH}$, centrifuged at $15000 \mathrm{rpm}$ for 5 min to

781 remove any particulates, and analyzed using HPLC-MS (Agilent Single Quad, column:

782 Poroshell 120 EC-C18 2.7um 4.6 x 50mm, flow rate $0.8 \mathrm{ml} / \mathrm{min}, 0.1 \%$ formic acid in

783 water (solvent A), 0.1\% formic acid in acetonitrile (solvent B), gradient: 1 min, 0.5\% B;

784 1-20 min, 0.5\%-100\% B; $20-25 \mathrm{~min}, 100 \%$ B). If drugs were deemed positive for MDM

785 in one or both of the two runs, they were analyzed a third time using both HPLC-MS and

786 HR-HPLC-MS/MS (Agilent QTOF, column: Poroshell 120 EC-C18 2.7um 2.1x100 mm,

787 flow rate $0.25 \mathrm{ml} / \mathrm{min}, 0.1 \%$ formic acid in water (solvent $\mathrm{A}$ ), $0.1 \%$ formic acid in

788 acetonitrile (solvent B), gradient: $1 \mathrm{~min}, 0.5 \% \mathrm{~B} ; 1-20 \mathrm{~min}, 0.5 \%-100 \% \mathrm{~B} ; 25-30 \mathrm{~min}$,

$789100 \%$ B). For selected molecules, cultures were scaled up and metabolites were

790 purified and their structures were elucidated using NMR (see below).

\section{Isolation and structural elucidation of selected metabolites}

$1 \mathrm{ml}$ of HD-1 glycerol stock was used to inoculate $100 \mathrm{ml}$ mGAM medium and

795 cultured for 24 hours at $37^{\circ} \mathrm{C}$ in an anaerobic chamber. After 24 hours, $2 \mathrm{ml}$ of $10 \mathrm{mM}$

796 capecitabine, hydrocortisone or nicardapine solutions were added to the HD-1 culture

797 and incubated for another 24 hours. After the second 24 hours, the cultures were 
798 extracted with double the volume of ethyl acetate and the organic solvent layer was

799 dried under vacuum in a rotary evaporator. The dried extract was then suspended in

$800 \mathrm{MeOH}$ and partitioned by reversed phase flash column chromatography (Mega Bond

801 Elut-C18 10g, Agilent Technology, USA) using the following mobile phase conditions:

802 solvent $A$, water with $0.01 \%$ formic acid; solvent $B$ acetonitrile with $0.01 \%$ formic acid,

803 gradient, $100 \%$ A to $100 \%$ B in $20 \%$ increments. Fractions containing the metabolites of

804 interest were identified by HPLC-MS, and reverse phase HPLC was used to purify each

805 metabolite using a fraction collector (Agilent Single Quad, column Poroshell 120 EC-

806 C18 2.7 um $4.6 \times 100 \mathrm{~mm}$, flow rate $0.8 \mathrm{ml} / \mathrm{min}, 0.1 \%$ formic acid in water (solvent $A$ ),

$8070.1 \%$ formic acid in acetonitrile (solvent B), gradient: $1 \mathrm{~min}, 0.5 \% \mathrm{~B} ; 1-30 \mathrm{~min}, 0.5 \%$ -

$808100 \%$ B; 30-35 min, 100\% B). The purified metabolites were subjected to NMR and HR-

809 MS/MS analysis. Structural elucidation details of capecitabine, hydrocortisone, and

810 nicardipine metabolites are detailed in Supplementary Data 1.

811

812

813

814

815

816

817

818

819

820

\section{MDM-Screen using a panel of representative isolates from the gut microbiome}

$3 \mathrm{ml}$ of pre-reduced medium (PYG, RCM, GAM, BHI or LB, depending on the isolate, incubated for 24 hours in the anaerobic chamber) was inoculated with the corresponding isolate's glycerol stock. Cultures were grown overnight at $37^{\circ} \mathrm{C}$ in an anaerobic chamber $\left(70 \% \mathrm{~N}_{2}, 25 \% \mathrm{CO}_{2}, 5 \% \mathrm{H}_{2}\right) .20 \mu \mathrm{L}$ of these seed cultures were inoculated into $3 \mathrm{ml}$ of the same selected medium, and incubated at $37^{\circ} \mathrm{C}$ under the same anaerobic conditions for an additional 24 hours. After 24 hours, $10 \mu \mathrm{L}$ of the 10 $\mathrm{mM}$ drug solution in DMSO, or of a DMSO control were added to the growing microbial culture and incubated for another 24 hours. In addition, $10 \mu \mathrm{L}$ of each drug were 
821 incubated for 24 hours under the same conditions in a no-bacterium, medium-only

822 control. After incubation, cultures were extracted with ethyl acetate and the organic

823 phase was dried under vacuum in a rotary evaporator. Extracts were suspended in 250

$824 \mu \mathrm{l}$ of $\mathrm{MeOH}$ and analyzed using HPLC-MS as described above.

825

826 TP and UP gene deletions in E. coli BW25113

827

E. coli BW25113 mutants that harbor a replacement of $d e o A$ or $u d p$ with a

828 kanamycin resistance gene were obtained from the Keio collection ${ }^{49}$. Since the

829 kanamycin resistance gene is flanked by FLP recognition target sites, we decided to

830 excise it and obtain in-frame deletion mutants. Plasmid pCP20, encoding the FLP

831 recombinase, was transformed to each of the mutants by electroporation, and

832 transformants were selected on Ampicillin at $30^{\circ} \mathrm{C}$ for 16 hours. 10 transformants from

833 each mutant were then picked in $10 \mu$ LB medium with no selection, and incubated at

$83442^{\circ} \mathrm{C}$ for 8 hours to cure them from the temperature-sensitive pCP20 plasmid. Each

835 growing colony was then streaked on three plates (LB-ampicillin, LB-kanamycin, and LB

836 with no selection). Mutants that could only grow on LB, but not on LB-ampicillin

837 (confirming the loss of the pCP20 plasmid), nor on LB-kanamycin (confirming the

838 excision of the kanamycin resistance gene) were confirmed to harbor the correct

839 deletion using PCR and DNA sequencing. Primers deoA-Check-F: 5'-

840 CGCATCCGGCAAAAGCCGCCTCATACTCTTTTCCTCGGGAGGTTACCTTG-3',

841 deoA-Check-R: 5'-

842 CAAATTTAAATGATCAGATCAGTATACCGTTATTCGCTGATACGGCGATA-3', udp-

843 Check-F: 5'- 
CGCGTCGGCCTTCAGACAGGAGAAGAGAATTACAGCAGACGACGCGCCGC-3',

845 and udp-Check-R: 5'-

846 TGTCTTTTTGCTTCTTCTGACTAAACCGATTCACAGAGGAGTTGTATATG-3’ were

847 used in PCR experiments to confirm the deletion of the deoA or upd genes and the

848 kanamycin resistance gene replacing them ${ }^{49}$. To construct the $\Delta d e o A / \Delta u d p$ double

849 knockout, the in-frame $\Delta u d p$ knockout obtained above was used as a starting point.

850 Plasmid pKD46 expressing the $\lambda$ Red recombinase was transformed to it using

851 electroporation, ${ }^{50}$ and transformants were selected on LB-Ampicillin at $30^{\circ} \mathrm{C}$ for 16

852 hours. One Ampicillin-resistant transformant was then cultured at $30^{\circ} \mathrm{C}$ in $50 \mathrm{ml}$ of LB-

853 Ampicillin, with an added $50 \mu$ of $1 \mathrm{M} \mathrm{L}$-arabinose to induce the expression of the

854 recombinase. At an optical density of 0.4-0.6, electrocompetent cells were prepared

855 from the growing culture by serial washes in ice cold $10 \%$ glycerol, and $\sim 300 \mathrm{ng}$ of a

856 linear PCR product were transformed to it by electroporation. This PCR product was

857 prepared by using the deoA-Check-F and deoA-Check-R primers on a template DNA

858 prepared from the $d e o A$ mutant of the Keio library, in which a kanamycin resistance

859 gene replaces deoA. After electroporation, transformants were selected on LB-

860 kanamycin at $37^{\circ} \mathrm{C}$ to induce the loss of the temperature sensitive pKD46 plasmid,

861 cultured in LB-kanamycin overnight at $37^{\circ} \mathrm{C}$, and checked by PCR to confirm the

862 correct recombination position. Finally, the kanamycin resistance gene was excised

863 from the deoA locus by the FLP recombinase using the same strategy explained above,

864 resulting in the final $\Delta d e o A / \Delta u d p$ mutant. 
Wild type E. coli BW25113, and corresponding TP knockout $(\Delta d e o A)$, UP knockout $(\Delta u d p)$, and TP/UP double knockout $(\Delta d e o A / \Delta u d p)$ strains were cultured overnight in LB medium (aerobically, shaking at $37^{\circ} \mathrm{C}, 50 \mathrm{ml}$ each). Triplicates of $3 \mathrm{ml}$

870 for each strain were incubated with $10 \mu \mathrm{l}$ of $10 \mathrm{mM}$ capecitabine (in DMSO) for an

871 additional 24 hours in an anaerobic chamber along with bacteria-only and media-only

872 controls. Cultures were then extracted and analyzed as previously described, except for 873 the addition of $20 \mu \mathrm{L}$ of $0.25 \mathrm{mg} / \mathrm{ml}$ of an internal standard (voriconazole) prior to the 874 extraction.

MDM-Screen of other FPs using wild type and mutant E. coli.

879 overnight in LB medium (aerobically, shaking at $37^{\circ} \mathrm{C}, 50 \mathrm{ml}$ each). Aliquots

$880(100 \mu \mathrm{l})$ of each strain were used to inoculate $3 \mathrm{ml}$ of M9 medium, which were grown

881 again overnight (aerobically, shaking at $37^{\circ} \mathrm{C}$ ). $10 \mu \mathrm{l}$ of $10 \mathrm{mM}$ doxifluridine (in DMSO)

882 or trifluridine (in methanol) were incubated with each culture for an additional 24 hours

883 in an anaerobic chamber, along with bacteria-only and medium-only controls. Cultures

884 were spun down and collected supernatants were lyophilized. The dried residues were

885 then resuspended in $500 \mu \mathrm{L}$ methanol and analyzed by HPLC-MS (Agilent Single Quad;

886 column: Poroshell 120 EC-C18 2.7um 4.6 x 100mm; flow rate: $0.6 \mathrm{ml} / \mathrm{min}$; solvent A:

$8870.1 \%$ formic acid in water: solvent B: $0.1 \%$ formic acid in acetonitrile) and the following

888 gradient: $1 \mathrm{~min}, 0.5 \% \mathrm{~B} ; 1-20 \mathrm{~min}, 0.5 \%-35 \% \mathrm{~B} ; 25-30 \mathrm{~min}, 35 \%-100 \% \mathrm{~B} ; 30-35 \mathrm{~min}$, $889100 \%$ B. 
891 Microbiome-dependent pharmacokinetic experiment

All animal experiments were conducted according to USA Public Health Service

893 Policy of Humane Care and Use of Laboratory Animals. All protocols were approved by

894 the Institutional Animal Care and Use Committee, protocol 2087-16 (Princeton

895 University). 8-10-weeks old (25-30 g) C57BL/6 mice were purchased from Jackson

896 laboratories. 12 mice were treated with a commonly used cocktail of antibiotics (1 g/l of

897 amplicilin, neomycin, metronidazole and $0.5 \mathrm{~g} / \mathrm{l}$ vancomycin) in drinking water for 14

898 days ${ }^{51}$. The antibiotic solution was supplemented with $5 \mathrm{~g} / \mathrm{l}$ aspartame to make it more

899 palatable ${ }^{52}$. During these two weeks, the gut microbiome composition was monitored by

900 collecting feces from each mouse and performing molecular and microbiological

901 analyses to make sure the microbiome is being cleared by the antibiotic treatment. On

902 day 15, no antibiotics are administered for 24 hours (a washout period). On day 16,

903 mice were separated into the two groups, 6 per group ( 3 males and 3 females). In group

9041 , mice remained non-colonized. In group 2, mice were administered $200 \mu$ l of freshly

905 thawed HD-1 glycerol stock using oral gavage. On day 17, the oral gavage was

906 repeated the same way to ensure the colonization of the administered bacteria (fecal

907 samples were collected on days 16 and 17 and cultured anaerobically to ensure

908 colonization). On day 18, the pharmacokinetic experiment was performed by monitoring

909 the fate of capecitabine in mouse blood and feces over time. A capecitabine dose

910 equivalent to a single human dose and adjusted to the weight of the mice was

911 administered by oral gavage (755 mg / $\mathrm{kg}$, as a solution in $50 \mu \mathrm{LMSO})$, then serial

912 sampling of tail vein blood (by tail snipping), as well as fecal collection were performed 
913 at these time points (zero, $20 \mathrm{~min}, 40 \mathrm{~min}, 60 \mathrm{~min}, 2$ hours, and 4 hours). Blood for each

914 time point $(30 \mu \mathrm{l})$ was collected using a $30 \mu$ l capillary tube and bulb dispenser

915 (Drummond Microcaps, Drummond Scientific), quickly dispensed in $60 \mu$ EDTA to

916 prevent blood coagulation, and stored on ice for up to 4 hours and then frozen at $-80^{\circ} \mathrm{C}$

917 until further analysis. Feces were also collected at the same time points (even though

918 defecation was left at will, we succeeded in collecting feces for most time points), stored

919 on ice for up to 4 hours and then frozen in $-80^{\circ} \mathrm{C}$ until further analysis. After the 4-hour

920 pharmacokinetic time point, mice were euthanized.

921 For chemical extraction, $2 \mu \mathrm{l}$ of an internal standard solution $(0.5 \mathrm{mg} / \mathrm{ml}$ of

922 voriconazole) were added to the blood / EDTA solution mentioned above, and the

923 sample was mixed using a vortex mixer. Next, $500 \mu \mathrm{l}$ of ethyl acetate was added and

924 mixed. The sample was then centrifuged briefly at $15000 \mathrm{rpm}$, and the organic layer

925 was transferred to a glass tube and evaporated under vacuum using rotary evaporation

926 (Speed Vac). The dried residue was dissolved in $100 \mu \mathrm{l}$ of $\mathrm{MeOH}$, and the solution was

927 centrifuged at $15000 \mathrm{rpm}$ and transferred to an autosampler vial for HR-HPLC-MS

928 analysis. For fecal samples, pellets were weighed (for later normalizations), and

929 suspended in $500 \mu$ l sterile Milli-Q water (Millipore Corporation, USA). $2 \mu$ l of an internal

930 standard solution $(0.5 \mathrm{mg} / \mathrm{ml}$ of voriconazole) were added to the sample, and the

931 mixtures were extracted with $500 \mu \mathrm{l}$ 1:1 ethyl acetate : $\mathrm{MeOH}$. Fecal debris were then

932 spun down and collected supernatants were dried under vacuum using a rotary

933 evaporator (Speed Vac). The dried residues were suspended in $100 \mu \mathrm{MeOH}$. The final

934 solutions were centrifuged at $15000 \mathrm{rpm}$ and transferred to autosampler vials. 
The prepared samples were analyzed by HR-HPLC-MS (Agilent QTOF).

936 Chromatography separation was carried out on a Poroshell 120 EC-C18 2.7 um 2.1 x

$937100 \mathrm{~mm}$ column (Agilent, USA) with the gradient: $99.5 \% \mathrm{~A}, 0.5 \% \mathrm{~B}$ to $100 \% \mathrm{~B}$ in 20

938 minutes and a flow rate of $0.25 \mathrm{ml} / \mathrm{min}$, where $A=0.1 \%$ formic acid in water and $B=$

$9390.1 \%$ formic acid in acetonitrile. A $10 \mu \mathrm{l}$ aliquot of the reconstituted extract was injected

940 into the HR-HPLC-MS system, and the Area Under the Curve (AUC) was integrated for

941 each metabolite and normalized by the internal standard's AUC. Peak identities were

942 confirmed by accurate mass, and by comparison of chromatographic retention time and

943 MS/MS spectra to those of authentic standards.

945 in vitro metabolism of capecitabine and deglycocapecitabine using human carboxyesterase 1

947 Human Carboxylesterase 1 (CES1) was purchased from Sigma-Aldrich. Capecitabine 948 or deglycocapecitabine $(3.25 \mu \mathrm{l}$ of a $10 \mathrm{mM}$ stock in DMSO) was added into CES (50 $949 \mu \mathrm{g})$ in $20 \mathrm{mM}$ HEPES pH 7.4; in a total volume of $150 \mu \mathrm{L}$ and then incubated at $37{ }^{\circ} \mathrm{C}^{53}$. 950 After $60 \mathrm{~min}$, the reaction was quenched with $150 \mu \mathrm{L}$ of acetonitrile and placed on ice.

951 The mixture was centrifuged for $3 \mathrm{~min}$ at $15000 \mathrm{rpm}$. The supernatant was dried under 952 vacuum using rotary evaporation (Speed Vac). The dried residues were suspended in $953200 \mu \mathrm{L} \mathrm{MeOH}$. The final solutions were centrifuged at $15000 \mathrm{rpm}$ and transferred to 954 autosampler vials. The samples were analyzed by HPLC-MS (Agilent Single Quad 955 6120) for metabolite formation (column: Poroshell 120 EC-C18 2.7um 4.6 x 50mm, flow

956 rate $0.8 \mathrm{ml} / \mathrm{min}, 0.1 \%$ formic acid in water (solvent $\mathrm{A}$ ), $0.1 \%$ formic acid in acetonitrile 957 (solvent B), gradient: $1 \mathrm{~min}, 0.5 \% \mathrm{~B} ; 1-30 \mathrm{~min}, 0.5 \%-100 \% \mathrm{~B} ; 30-35 \mathrm{~min}, 100 \% \mathrm{~B}$ ). 
959 Data availability

All data reported in this study are included in this manuscript and accompanying

961 Supplementary Information.

\section{Acknowledgments}

We would like to thank Wei Wang and the Lewis Sigler Institute sequencing core

965 facility for assistance with high-throughput 16S rRNA gene amplicon sequencing,

966 Matthew Cahn for assistance with sequencing data analysis, Joseph Koos, A. James

967 Link, and Yuki Sugimoto for assistance with Mass Spectrometry, Riley Skeen-Gaar for

968 assistance with statistical analysis, Joseph Sheehan and Zemer Gitai for assistance

969 with obtaining the Keio library mutants, Laboratory Animal Resources at Princeton

970 University for assistance with mouse studies, and members of the Donia lab for useful

971 discussions. Funding for this project has been provided by an Innovation Award from

972 the Department of Molecular Biology, Princeton University, and an NIH Director's New

973 Innovator Award (ID: 1DP2AI124441), both to M.S.D. B.J. is funded by a New Jersey

974 Commission on Cancer Research Pre-doctoral award (ID: DFHS18PPC056), and J.L is

975 funded by a National Science Foundation Graduate Research Fellowship (ID:

976 2017249408).

977

\section{Author contributions}


980 performed experiments and analyzed the data. M.S.D., P.C., B.J., and J.L. wrote the

981 manuscript.

982

983 Competing financial interests

The authors declare no competing financial interests.

985

986 Supplementary Information

988 provided.

989

990

991

992

993

994

995

996

997

998

999

1000

1001

1002 


\section{REFERENCES}

1004

1005

1006

1007

1008

1009

1010

1011

1012

1013

1014

1015

1016

1017

1018

1019

1020

1021

1022

1023

1024

1025

1026

1027

1028

1029

1030

1031

1032

1033

1034

1035

1036

1037

1038

1039

1040

1041

1042

1043

1044

1045

$1 \quad$ Kimura, T. et al. Drug absorption from large intestine: physicochemical factors governing drug absorption. Biol. Pharm. Bull. 17, 327-333 (1994).

$2 \mathrm{Li}, \mathrm{H}$. \& Jia, W. Cometabolism of microbes and host: implications for drug metabolism and drug-induced toxicity. Clin. Pharmacol. Ther. 94, 574-581, doi:10.1038/clpt.2013.157 (2013).

3 Falony, G. et al. Population-level analysis of gut microbiome variation. Science 352, 560564, doi:10.1126/science.aad3503 (2016).

$4 \quad$ Clayton, T. A., Baker, D., Lindon, J. C., Everett, J. R. \& Nicholson, J. K. Pharmacometabonomic identification of a significant host-microbiome metabolic interaction affecting human drug metabolism. Proc. Natl. Acad. Sci. U S A 106, 1472814733, doi:10.1073/pnas.0904489106 (2009).

5 lida, N. et al. Commensal bacteria control cancer response to therapy by modulating the tumor microenvironment. Science 342, 967-970, doi:10.1126/science.1240527 (2013).

6 Sivan, A. et al. Commensal Bifidobacterium promotes antitumor immunity and facilitates anti-PD-L1 efficacy. Science 350, 1084-1089, doi:10.1126/science.aac4255 (2015).

7 Vetizou, M. et al. Anticancer immunotherapy by CTLA-4 blockade relies on the gut microbiota. Science 350, 1079-1084 (2015).

$8 \quad$ Wallace, B. D. et al. Alleviating cancer drug toxicity by inhibiting a bacterial enzyme. Science 330, 831-835, doi:10.1126/science.1191175 (2010).

9 Meinl, W., Sczesny, S., Brigelius-Flohe, R., Blaut, M. \& Glatt, H. Impact of gut microbiota on intestinal and hepatic levels of phase 2 xenobiotic-metabolizing enzymes in the rat.

Drug Metab. Dispos. 37, 1179-1186, doi:10.1124/dmd.108.025916 (2009).

10 Qin, J. et al. A human gut microbial gene catalogue established by metagenomic sequencing. Nature 464, 59-65, doi:10.1038/nature08821 (2010).

11 Backhed, F., Ley, R. E., Sonnenburg, J. L., Peterson, D. A. \& Gordon, J. I. Host-bacterial mutualism in the human intestine. Science 307, 1915-1920, doi:10.1126/science.1104816 (2005).

12 Ilett, K. F., Tee, L. B., Reeves, P. T. \& Minchin, R. F. Metabolism of drugs and other xenobiotics in the gut lumen and wall. Pharmacol. Ther. 46, 67-93 (1990).

13 Scheline, R. R. Metabolism of foreign compounds by gastrointestinal microorganisms. Pharmacol. Rev. 25, 451-523 (1973).

14 Spanogiannopoulos, P., Bess, E. N., Carmody, R. N. \& Turnbaugh, P. J. The microbial pharmacists within us: a metagenomic view of xenobiotic metabolism. Nat. Rev. Microbiol. 14, 273-287, doi:10.1038/nrmicro.2016.17 (2016).

15 Lloyd-Price, J. et al. Strains, functions and dynamics in the expanded Human Microbiome Project. Nature 550, 61-66, doi:10.1038/nature23889 (2017).

16 Callahan, B. J. et al. DADA2: High-resolution sample inference from Illumina amplicon data. Nat. Methods 13, 581-583, doi:10.1038/nmeth.3869 (2016).

17 Bolyen, E. et al. QIIME 2: Reproducible, interactive, scalable, and extensible microbiome data science. PeerJ Preprints 6, e27295v27292, doi:10.7287/peerj.preprints.27295v2 (2018). 
104618 Bokulich, N. A. et al. Optimizing taxonomic classification of marker-gene amplicon sequences with QIIME 2's q2-feature-classifier plugin. Microbiome 6, 90,

1048

1049 doi:10.1186/s40168-018-0470-z (2018).

1050

1051

1052

1053

1054

1055

19 McDonald, D. et al. An improved Greengenes taxonomy with explicit ranks for ecological and evolutionary analyses of bacteria and archaea. ISME J. 6, 610-618,

1056

1057 doi:10.1038/ismej.2011.139 (2012).

20 Rettedal, E. A., Gumpert, H. \& Sommer, M. O. Cultivation-based multiplex phenotyping of human gut microbiota allows targeted recovery of previously uncultured bacteria. Nat. Commun. 5, 4714, doi:10.1038/ncomms5714 (2014).

21 McDonald, D. et al. American Gut: an Open Platform for Citizen Science Microbiome Research. mSystems 3, doi:10.1128/mSystems.00031-18 (2018).

22 Tramontano, M. et al. Nutritional preferences of human gut bacteria reveal their

1058 metabolic idiosyncrasies. Nat. Microbiol. 3, 514-522, doi:10.1038/s41564-018-0123-9

1059

1060 (2018).

23 Maier, L. et al. Extensive impact of non-antibiotic drugs on human gut bacteria. Nature

1061 555, 623-628, doi:10.1038/nature25979 (2018).

106224 Peppercorn, M. A. \& Goldman, P. The role of intestinal bacteria in the metabolism of

1063 salicylazosulfapyridine. J. Pharmacol. Exp. Ther. 181, 555-562 (1972).

1064

Azadkhan, A. K., Truelove, S. C. \& Aronson, J. K. The disposition and metabolism of

1065

1066 sulphasalazine (salicylazosulphapyridine) in man. Br. J. Clin. Pharmacol. 13, 523-528 (1982).

1067

26 Kuroiwa, M., Inotsume, N., Iwaoku, R. \& Nakano, M. Reduction of Dantrolene by Enteric

1068

1069 Bacteria. YAKUGAKU ZASSHI 105, 770-774, doi:10.1248/yakushi1947.105.8_770 (1985).

1070

1071

1072

Elmer, G. W. \& Remmel, R. P. Role of the intestinal microflora in clonazepam metabolism in the rat. Xenobiotica 14, 829-840, doi:10.3109/00498258409151481 (1984).

1073

1074

Meuldermans, W. et al. The metabolism and excretion of risperidone after oral administration in rats and dogs. Drug Metab. Dispos. 22, 129 (1994).

1075 Mannens, G. et al. Absorption, metabolism, and excretion of risperidone in humans.

1076 Drug Metab. Dispos. 21, 1134-1141 (1993).

1077

1078

30 Fedorowski, T., Salen, G., Tint, G. S. \& Mosbach, E. Transformation of chenodeoxycholic acid and ursodeoxycholic acid by human intestinal bacteria. Gastroenterology 77, 10681073 (1979).

1079

1080

1081

1082

1083

1084

1085

1086

1087

Ridlon, J. M. et al. Clostridium scindens: a human gut microbe with a high potential to convert glucocorticoids into androgens. J. Lipid Res. 54, 2437-2449, doi:10.1194/jlr.M038869 (2013).

32 Devendran, S., Mendez-Garcia, C. \& Ridlon, J. M. Identification and characterization of a 20beta-HSDH from the anaerobic gut bacterium Butyricicoccus desmolans ATCC 43058. J. Lipid Res. 58, 916-925, doi:10.1194/jlr.M074914 (2017).

33 Northfield, T. C. \& McColl, I. Postprandial concentrations of free and conjugated bile acids down the length of the normal human small intestine. Gut 14, 513-518 (1973).

34 Lamont, E. B. \& Schilsky, R. L. The oral fluoropyrimidines in cancer chemotherapy. Clin. 1088 Cancer Res. 5, 2289-2296 (1999). 
108935 Longley, D. B., Harkin, D. P. \& Johnston, P. G. 5-fluorouracil: mechanisms of action and

1090

1091

1092

1093

1094

1095

1096

1097

1098

1099

1100

1101

1102

1103

1104

1105

1106

1107

1108

1109

1110

1111

1112

1113

1114

1115

1116

1117

1118

1119

1120

1121

1122

1123

1124

1125

1126

1127

1128

1129

1130

1131

1132

clinical strategies. Nat. Rev. Cancer 3, 330-338, doi:10.1038/nrc1074 (2003).

36 Cleary, J. M. et al. A phase 1 study of the pharmacokinetics of nucleoside analog trifluridine and thymidine phosphorylase inhibitor tipiracil (components of TAS-102) vs trifluridine alone. Invest. New Drugs 35, 189-197, doi:10.1007/s10637-016-0409-9 (2017).

37 Zampino, M. G. et al. Pharmacokinetics of oral doxifluridine in patients with colorectal cancer. Tumori 85, 47-50 (1999).

38 Nakayama, H. et al. Intestinal anaerobic bacteria hydrolyse sorivudine, producing the high blood concentration of 5-(E)-(2-bromovinyl)uracil that increases the level and toxicity of 5-fluorouracil. Pharmacogenetics 7, 35-43 (1997).

39 Temmink, O. H. et al. Activity and substrate specificity of pyrimidine phosphorylases and their role in fluoropyrimidine sensitivity in colon cancer cell lines. Int. J. Biochem. Cell Biol. 39, 565-575, doi:10.1016/j.biocel.2006.10.009 (2007).

40 Lenz, H. J., Stintzing, S. \& Loupakis, F. TAS-102, a novel antitumor agent: a review of the mechanism of action. Cancer Treat. Rev. 41, 777-783, doi:10.1016/j.ctrv.2015.06.001 (2015).

41 Kim, N. K. et al. Intravenous 5-Fluorouracil Versus Oral Doxifluridine as Preoperative Concurrent Chemoradiation for Locally Advanced Rectal Cancer: Prospective Randomized Trials. Jpn. J. Clin. Oncol. 31, 25-29, doi:10.1093/jjco/hye009 (2001).

42 Min, J. S., Kim, N. K., Park, J. K., Yun, S. H. \& Noh, J. K. A prospective randomized trial comparing intravenous 5-fluorouracil and oral doxifluridine as postoperative adjuvant treatment for advanced rectal cancer. Ann. Surg. Oncol. 7, 674-679 (2000).

43 Reigner, B., Blesch, K. \& Weidekamm, E. Clinical pharmacokinetics of capecitabine. Clin. Pharmacokinet. 40, 85-104, doi:10.2165/00003088-200140020-00002 (2001).

44 Tabata, T., Katoh, M., Tokudome, S., Nakajima, M. \& Yokoi, T. Identification of the cytosolic carboxylesterase catalyzing the 5'-deoxy-5-fluorocytidine formation from capecitabine in human liver. Drug Metab. Dispos. 32, 1103-1110, doi:10.1124/dmd.104.000554 (2004).

45 Quinney, S. K. et al. Hydrolysis of capecitabine to 5'-deoxy-5-fluorocytidine by human carboxylesterases and inhibition by loperamide. J. Pharmacol. Exp. Ther. 313, 10111016, doi:10.1124/jpet.104.081265 (2005).

46 Haiser, H. J. et al. Predicting and manipulating cardiac drug inactivation by the human gut bacterium Eggerthella lenta. Science 341, 295-298, doi:10.1126/science.1235872 (2013).

47 Goodman, A. L. et al. Extensive personal human gut microbiota culture collections characterized and manipulated in gnotobiotic mice. Proc. Natl. Acad. Sci. U S A 108, 6252-6257, doi:10.1073/pnas.1102938108 (2011).

48 Caporaso, J. G. et al. Ultra-high-throughput microbial community analysis on the Illumina HiSeq and MiSeq platforms. ISME J. 6, 1621-1624, doi:10.1038/ismej.2012.8 (2012).

49 Baba, T. et al. Construction of Escherichia coli K-12 in-frame, single-gene knockout mutants: the Keio collection. Mol. Syst. Biol. 2, 2006 0008, doi:10.1038/msb4100050 (2006). 
113350 Datsenko, K. A. \& Wanner, B. L. One-step inactivation of chromosomal genes in Escherichia coli K-12 using PCR products. Proc. Natl. Acad. Sci. U S A 97, 6640-6645, doi:10.1073/pnas.120163297 (2000).

51 Planer, J. D. et al. Development of the gut microbiota and mucosal IgA responses in twins and gnotobiotic mice. Nature 534, 263-266, doi:10.1038/nature17940 (2016).

52 Karmarkar, D. \& Rock, K. L. Microbiota signalling through MyD88 is necessary for a systemic neutrophilic inflammatory response. Immunology 140, 483-492, doi:10.1111/imm.12159 (2013).

1141

53 Hatfield, M. J. et al. Biochemical and molecular analysis of carboxylesterase-mediated hydrolysis of cocaine and heroin. Br. J. Pharmacol. 160, 1916-1928, doi:10.1111/j.14765381.2010.00700.x (2010). 